\title{
DISEÑO DE UN DISPOSITIVO PARA AUTOREHABILITACIÓN PASIVA DE RODILLA
}

\author{
José Segnini1*; Anderson Chagna ${ }^{1}$; Mary Vergara ${ }^{1}$ \\ ${ }^{1}$ Pontificia Universidad Católica de Ecuador. Sede Ibarra, Escuela de Diseño, Grupo de Investigación en Diseño Sustentable \\ GIDISUS, Ibarra-Ecuador
}

*Autor para correspondencia: e-mail: jmsegnini@pucesi.edu.ec

Recibido: 2017/15/08 Aprobado: 2018/13/06

D0I: https://doi.org/10.26621/XIV18.2018.06.A08.PUCESI.2550.6684

\section{RESUMEN}

Para el 2016 en Ecuador se encuentran registradas 193.520 personas con discapacidad física, de éstas 9.895 poseen daños patológicos en la rodilla, lo que representa un alto porcentaje de la población que necesita rehabilitación de rodilla y que, en su primera etapa, es posible que el paciente no pueda realizar esfuerzos sobre la misma. Así, para tratar este tipo de afecciones es común el uso de diversos dispositivos automatizados, que generalmente son importados, lo que implica un alto costo asociado al producto. De allí, surge la necesidad de realizar un diseño conceptual de un rehabilitador de rodilla pasivo, con entrada motriz dada por el usuario, integrado por un mecanismo de geometría sencilla, construido con materiales que se encuentren en el país y con el menor costo posible. En la investigación realizada se plantea un análisis jerárquico de alternativas para seleccionar aquella que cumple globalmente con los criterios mencionados y que permitan desarrollar movimientos de flexión y extensión. Además, se toma en cuenta la parte estética y las necesidades del producto, apoyada en las indicaciones e información recolectada con fisioterapeutas y orientada a la autorehabilitación de la población adulta de Ecuador. Los resultados obtenidos, muestran que se puede materializar un diseño en el que se realiza un análisis cinemático y de resistencia de materiales, utilizando programas CAD-CAE que sirven para definir totalmente la geometría y materiales del dispositivo en base a un mecanismo de cuatro barras, el cual permite realizar movimientos de flexo-extensión de $170^{\circ}$ a $90^{\circ}$ que corresponden a las fases de rehabilitación de la rodilla. Además, se incorporan formas exteriores con una cromática dispuesta con colores que despiertan y estimulan a realizar la actividad de rehabilitación, estos colores armonizan el diseño con la orientación del producto.

Palabras Claves: Dispositivo; rodilla; rehabilitación pasiva, tratamiento terapéutico

\begin{abstract}
During 2016, 408,021 people with disabilities have been registered in Ecuador, from which 193,520 are physically handicapped and 9,895 of them have pathological damage in the knee, which evidences there is a high percentage of the population that needs knee rehabilitation, a problem that, in its first stage, might cause the patient is not be able to make any efforts on the knee.

In order to treat this type of conditions, the use of various automated devices is common. Said devices are usually imported, which implies a high cost associated with the product. From this, it arises the need to make a conceptual design of a passive knee rehabilitation with a user's input, composed of a simple geometry mechanism, built with materials found in the country and at the lowest possible cost. In this
\end{abstract}


research, there is a hierarchical analysis of alternatives to select the one that complies globally with the aforementioned criteria and that allow to develop flexion and extension movements. It is also taken into account the aesthetic part and the needs of the product supported by the indications and the information collected with physiotherapists and oriented to the self-rehabilitation of the adult population of Ecuador The results have shown that is possible to materialize a design which combines a cinematic analysis and resistance of materials using CAD-CAE programs that serve to completely define the geometry and materials of the device based on a mechanism of four bars, which allows flexion-extension movements of $170^{\circ}$ to $90^{\circ}$ that correspond to the knee rehabilitation phases. Also, it incorporates exterior forms with a chromatic set with colors that awaken and stimulate the activity of rehabilitation, these colors harmonize design with the orientation of the product.

Keywords: Device, knee, passive rehabilitation, therapy treatment.

\section{INTRODUCCIÓN}

La rodilla es una articulación importante del cuerpo humano ya que, gracias a sus funciones, permite ejecutar múltiples actividades que incluyen movimientos de flexión y extensión, donde prácticamente no existe una actividad que implique desplazamiento en la cual no se utilice ésta. Lo anterior, implica un decremento de la calidad de vida cuando el cuerpo humano sufre luxaciones, esguinces, torceduras de articulaciones, ligamentos de la rodilla, y otras enfermedades asociadas a la misma, acompañadas con accidentes viales, laborales o por deporte que pueden culminar o no con una intervención quirúrgica y cuyo primer tratamiento requiere de una rehabilitación pasiva de rodilla, donde una persona o dispositivo moviliza las extremidades sin ningún esfuerzo del paciente, así que la articulación se somete a un rango de movimiento angular preestablecido y por un período de tiempo determinado.

Para el 2016 en Ecuador se encuentran registradas 193.520 personas con discapacidad física (CONADIS, 2016), de éstas 9.895 poseen daños patológicos en la rodilla (INEC, 2016), lo que representa un alto porcentaje de la población que necesita rehabilitación de rodilla. Esta situación, es cada vez más preocupante, debido principalmente a que el costo de equipos especializados es muy alto.

En el mercado, existe una cantidad importante de dispositivos que realizan rehabilitación de rodilla de forma autónoma, de tal manera que la mayoría pueden agruparse en dispositivos con control de posición y dinámicos que pueden utilizar neumática, resortes lineales, hidráulica y barras torsionales para controlar la articulación de la rodilla como se muestra en (Wilkening et al., 2012; Tian, 2015; Umchid y Taraphongphan, 2016) donde, además se especifica que existe una necesidad de realizar investigaciones direccionadas a que los dispositivos diseñados se asemejen más a realizar la función normal de la rodilla durante todo el ciclo de andar. También (Swortec, 2017) presenta a MotionMaker TM que es un sistema de entrenamiento programable para los miembros inferiores desarrollado por (Schmitt et al., 2004) que tiene tres grados de libertad, ver también (Guzmán et al., 2014) y está compuesto por dos ortesis además de motores, sensores y una unidad de control que gestiona la simulación eléctrica con regulaciones en tiempo real.

En esta misma dirección, en los últimos años, se han presentado varias patentes que muestran la evolución de dichos dispositivos que consideran varias formas y configuraciones geométricas y que van desde sistemas solamente mecánicos actuados por el usuario, hasta los controlados automáticamente, ver por ejemplo (Xiaoning et al., 2010; Ding et al., 2016; Branch et al., 2016). Por esto, se observa que los movimientos de rehabilitación pueden ser realizados de manera autónoma por el paciente, así Hall (Hall, 2017) presenta un sistema con entrada motriz dada por el usuario, que posee un cierto grado de movilidad. Sin embargo, algunos pacientes pueden requerir diferentes extensiones y flexiones, debido al grado de inmovilización que sufre la rodilla y, por lo tanto, es importante desarrollar mecanismos que sean capaces de cubrir esta necesidad, como el presentado en (Vergara et al, 2017).

En esta investigación, se presenta un diseño conceptual de un rehabilitador de rodilla con entrada motriz dada por el usuario, con restricciones asociadas al uso de geometrías sencillas, material disponible en la 
zona y de bajo costo, que contribuye con la progresiva rehabilitación sin supervisión médica. Para esto, en el proceso de diseño, primero se presentan alternativas de mecanismos, formas y materiales para seleccionar las más adecuadas según los requerimientos planteados. Posteriormente, utilizando programas CAD- CAE se realizan simulaciones numéricas validadas para definir un rehabilitador de rodilla de aluminio con mecanismo de manivela, biela corredera con un grado de libertad, capaz de soportar cargas máximas dadas por el paciente sin deformarse permanentemente. Además, se han utilizado formas exteriores con una cromática dispuesta con colores que despierten y estimulan a realizar la actividad de rehabilitación, rompiendo con lo regular de las propuestas clásicas que dejan atrás los colores blancos, grises y tonos obscuros.

\section{MATERIALES Y MÉTODOS}

Para desarrollar el diseño del rehabilitador de rodilla, es importante definir los requerimientos que están asociados a varios aspectos de la información aportada por expertos en el área de rehabilitación, donde se despliegan los datos necesarios y suficientes para la conceptualización y proceso de diseño, que se lleva a cabo en varias etapas considerando el uso de herramientas computacionales que introduce en este proceso no sólo la estética, si no, el cálculo en ingeniería para validar los materiales y formas utilizadas para el dispositivo. De esta forma, se desarrolla un proceso de diseño que involucra el método de Bruce Archer (2015) y Karim Rashid (2017) con una combinación propia que incluye el comportamiento estructural del dispositivo, considerando, además el entorno del dispositivo rehabilitador de rodilla, que debe incluir restricciones asociadas a los costos. Por lo tanto, los elementos que comprenden el esquema proyectual desarrollado, contiene todos los elementos mostrados en la figura 1 que lleva a plantear que, el mecanismo diseñado es de tipo manual para la auto-rehabilitación con un público objetivo que corresponde a los adultos de 18 a 20 años de edad.

Una parte importante a estudiar, una vez que se ha definido y seleccionado de varias alternativas del mecanismo, la forma y el material, se añaden las restricciones y el estado de carga, para realizar la simulación numérica considerando la posición crítica del mecanismo obtenida con el programa comercial working model. Dicha simulación para el análisis estructural está hecha con el método de elementos finitos y se sigue un algoritmo simple dentro del proceso del diseño modificado de (Vergara et al, 2017) como se muestra en la figura 2 y en el cuál se contemplan los pasos desde la concepción del problema, embebido en el entorno, hasta la construcción del prototipo. Así, el primer paso consiste en reconocer el problema desde su propio entorno, pasando por una etapa de solución que considera el proceso de creatividad, investigación preliminar, soluciones similares existentes, junto con las herramientas tecnológicas disponibles para presentar bocetos y seleccionar alguno, hasta pasar a la simulación del rehabilitador, considerando normativa para dispositivos biomecánicos con su respectiva antropometría y ergonomía.

Figura 1. Esquema Proyectual de Dispositivo Rehabilitador de Rodilla

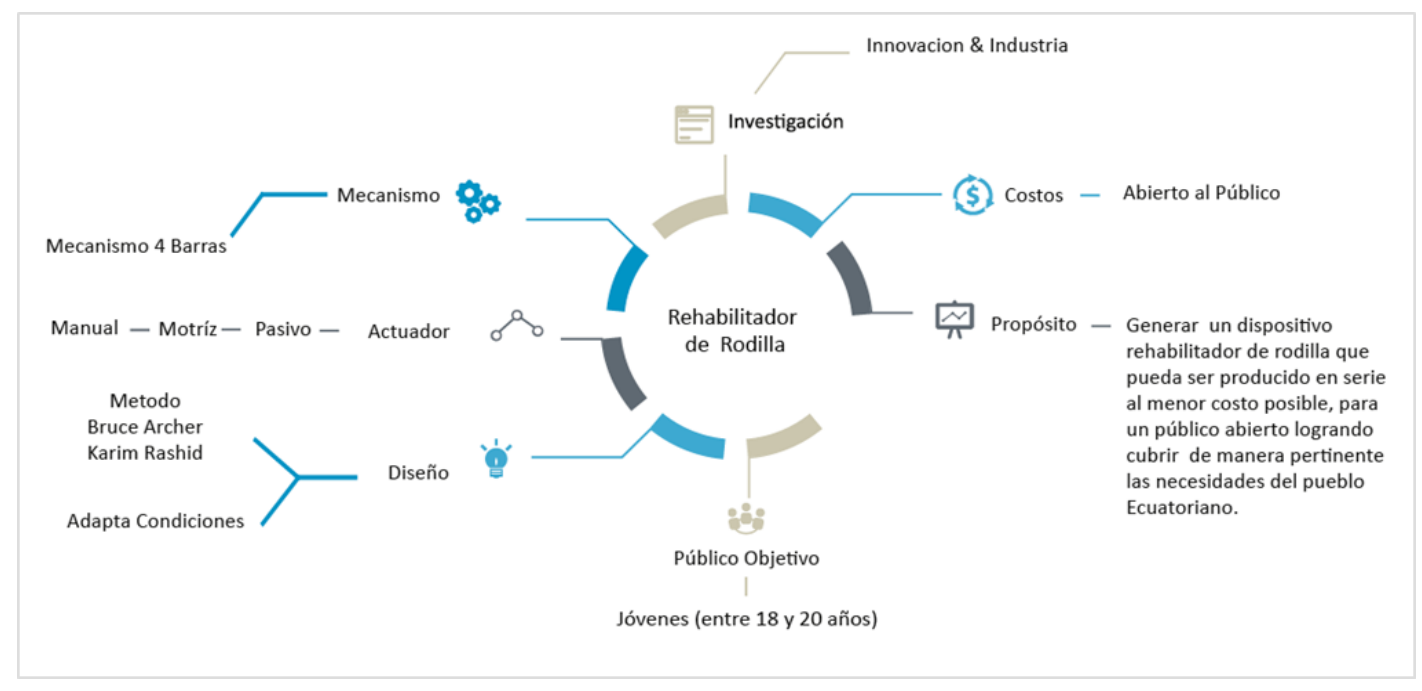


Para la obtención de la geometría final, se utiliza el programa Autodesk Inventor y su plataforma de simulación, que involucra el preproceso donde se definen: la geometría y las condiciones de carga, sujeción y material, el proceso para determinar esfuerzos, desplazamiento, deformaciones y factor de seguridad y postproceso para visualizar los resultados que sirven para verificar que el sistema es capaz de soportar las cargas máximas sin deformarse permanentemente y así pasar al diseño de detalle.

\section{RESULTADOS Y DISCUSIÓN}

Para la selección de mecanismos, formas y materiales se sigue un análisis jerárquico necesario con el programa Expert Choice, donde se definen criterios que serán utilizados para seleccionar las alternativas.

\section{Análisis jerárquico para selección de Alternativas}

En el esquema de proceso de diseño propuesto, se establecen alternativas para mecanismos, forma externa y materiales eficaces para el rehabilitador. En este contexto, se presentan diferentes alternativas dirigidas al rehabilitador de rodilla, las cuales son sometidas a evaluación y cuyos criterios y función objetivo se detallan en la figura 3.

Figura 2. Algoritmo de solución para realizar la simulación numérica

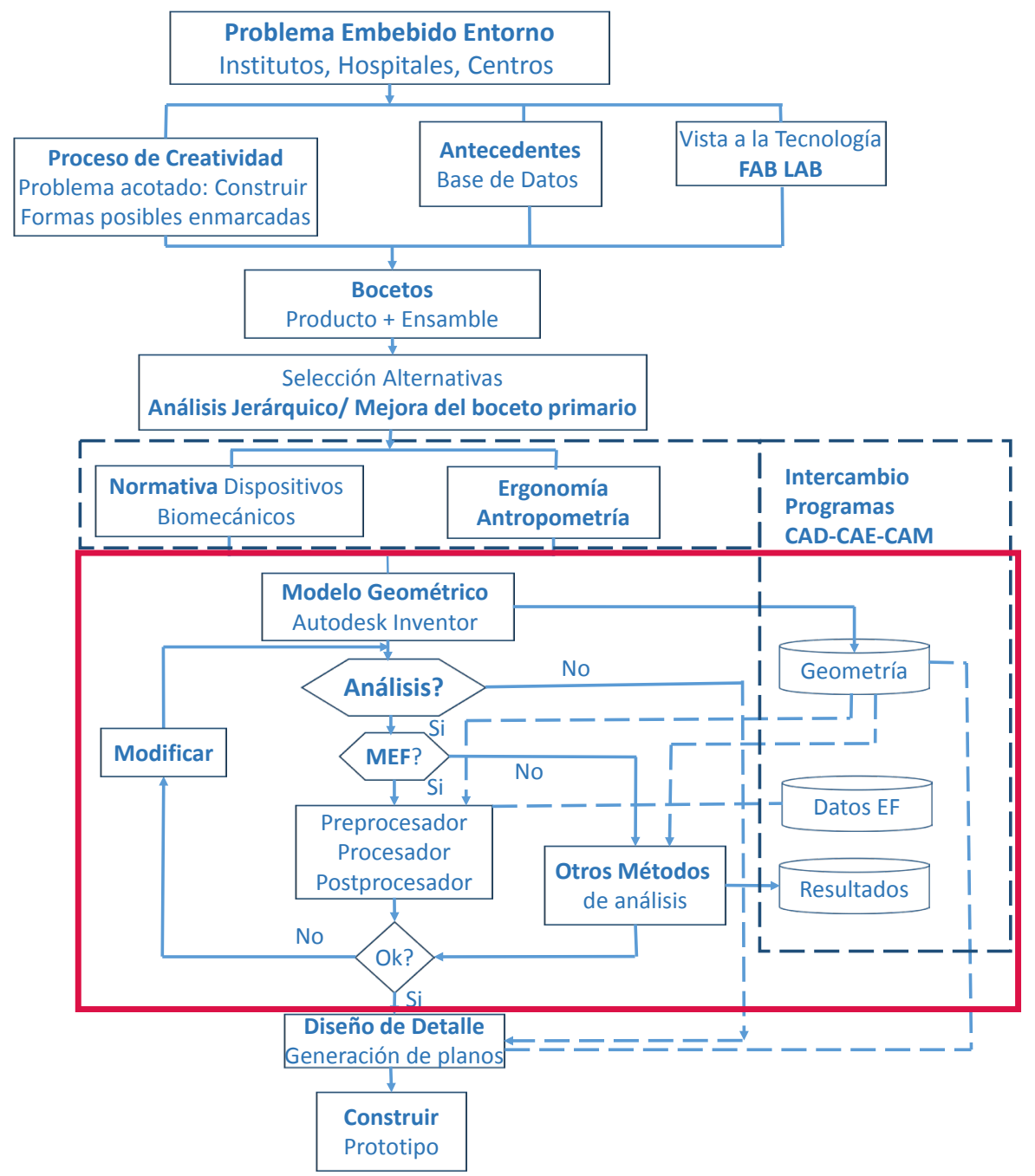


Figura 3. Función Jerárquica para Mecanismo, forma y material del rehabilitador

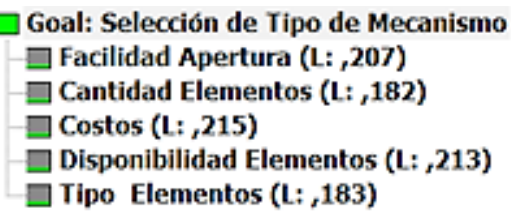

\author{
Goal: Selección materia prima \\ $\square$ Maleabilidad (L: ,167) \\ $\square$ Costos (L: , 178) \\ $\square$ Resistencia (L: ,169) \\ $\square$ Peso (L: , 155) \\ $\square$ Tecnologia para trabajar (L: ,164) \\ $\square$ Beneficio médico $(1:$, 167)
}

Para el planteamiento preliminar del diseño de la forma, se exponen, como base alternativa geométricas orgánicas que concilian el concepto de diseño y se asocian al mobiliario ergonómico contemporáneo, para ello se consideran mecanismos que cumplen con las restricciones de movimiento asociadas a las fases de rehabilitación de la rodilla que se ajustan a la forma, función y composición del mecanismo, buscando perfeccionar la compatibilidad con dicho mecanismo.

En la figura 4, se observa que los mecanismos 1 y 2 están compuestos por engranajes que transmiten el movimiento giratorio, desde uno de sus extremos, para transformarlo en movimiento lineal desde un soporte central que agrega un sistema graduado de flexión y extensión sobre el soporte central.

Figura 4. Bocetos del mecanismo 1 y 2

MECANISMO 12 Engranes, Cadena, Manivela

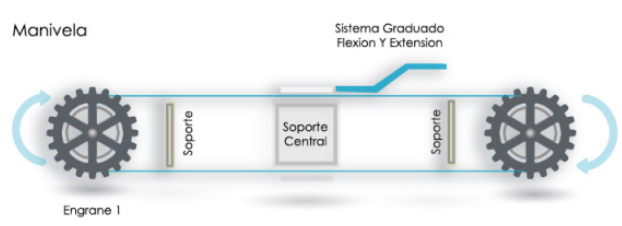

MECANISMO 2 Engranes cònicos, Biela, Manivela

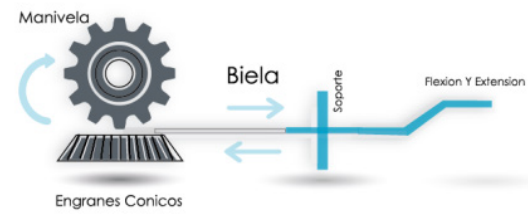

Los mecanismos 3 y 4, mostrados en la figura 5, utilizan un pistón que se conecta directamente a un componente corredizo que convierte el movimiento lineal en movimiento angular, que proporciona los movimientos de flexión y extensión.

Figura 5. Bocetos del mecanismo 3 y 4

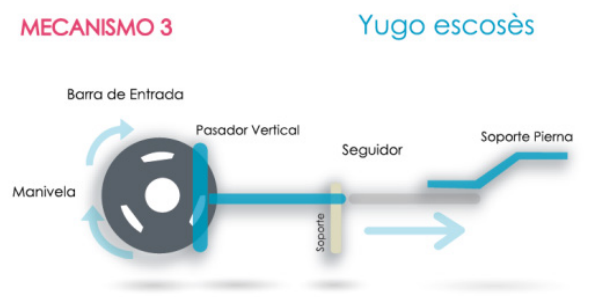

MECANISMO 4 Manivela, Biela, Pistòn

Los mecanismos 5 y 6 corresponden a mecanismos compuestos por barras que transforman un movimiento angular en uno lineal, que cumple la misma función, de los anteriores, de generar la flexión y extensión necesaria para la rehabilitación (ver figura 6). 
Figura 6. Bocetos del mecanismo 5 y 6
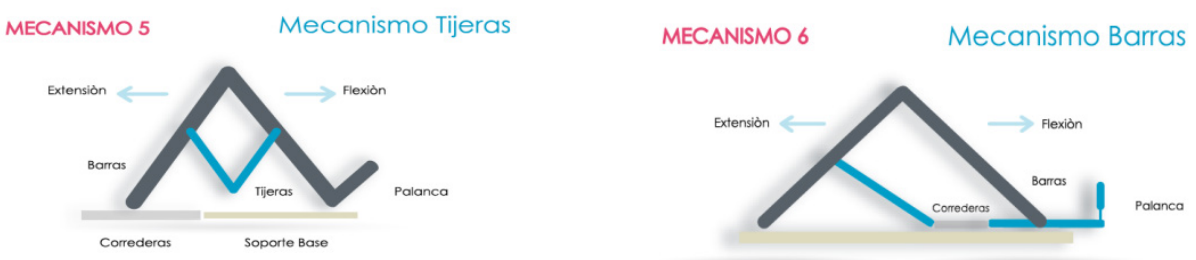

La alternativa del mecanismo 6 resulta la opción más viable para el diseño, de acuerdo a la vialidad de construcción, facilidad de obtención de material y el nivel general para el modo de trabajo. Así, el mecanismo 6 tiene el mayor puntaje, de acuerdo a cada uno de los criterios, como se presenta en la figura 7

Figura 7. Evaluación de las alternativas de los mecanismos

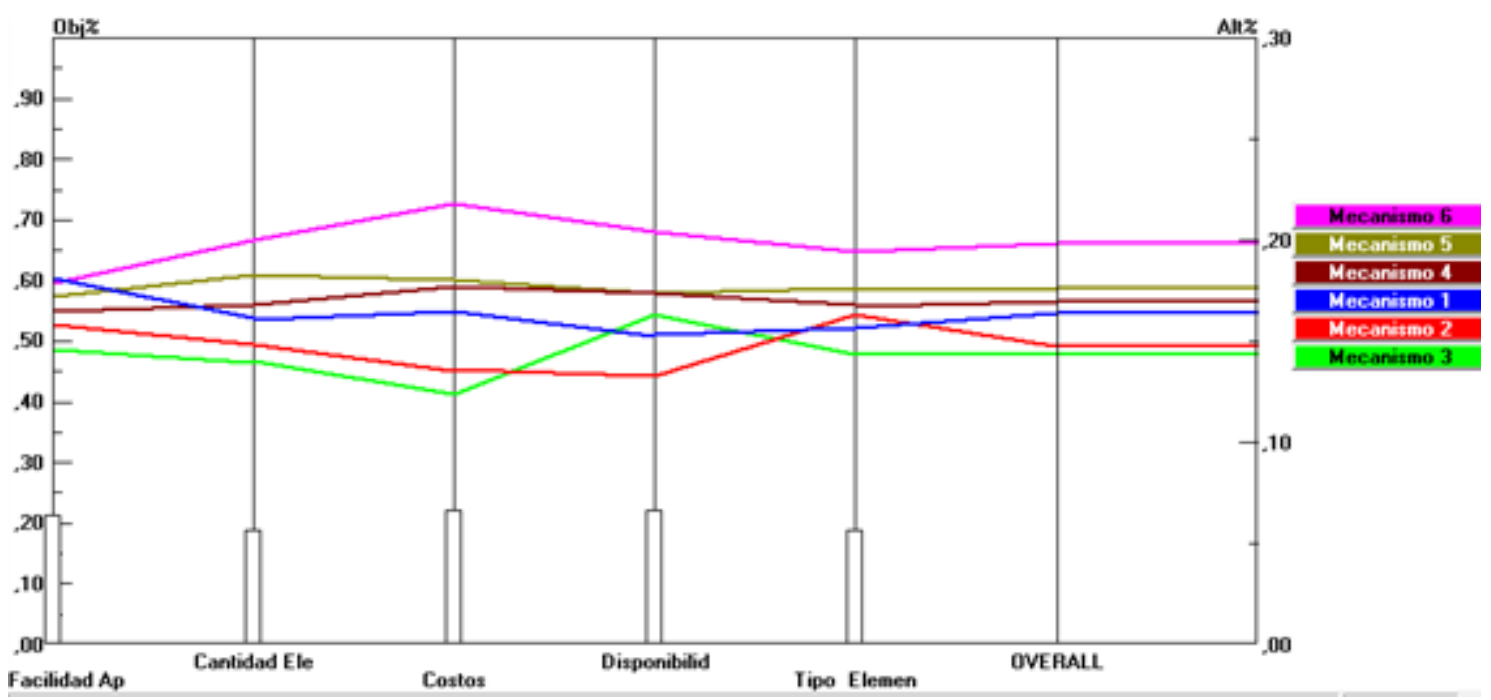

Los bocetos de las formas exteriores tienen una cromática dispuesta con colores, que despierten y estimulen a realizar la actividad de rehabilitación, corresponden a cinco propuestas, donde su desarrollo se detalla en los bocetos presentados en las figuras, desde la 8 a la 12

Figura 8. Proceso de diseño de la Forma 1

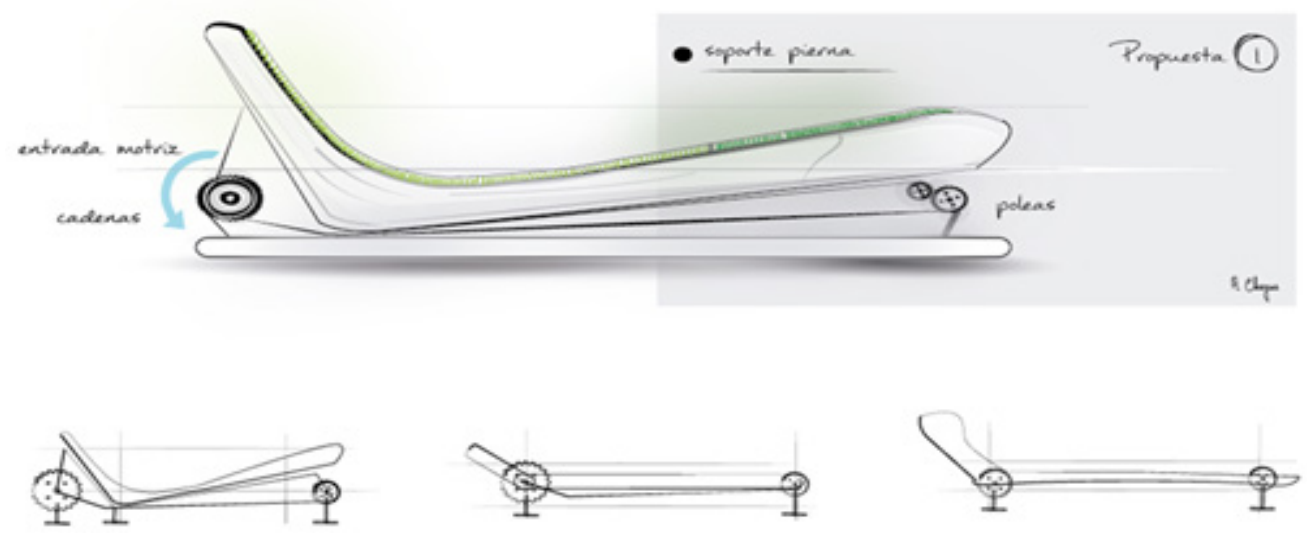


Figura 9. Proceso de diseño de la Forma 2
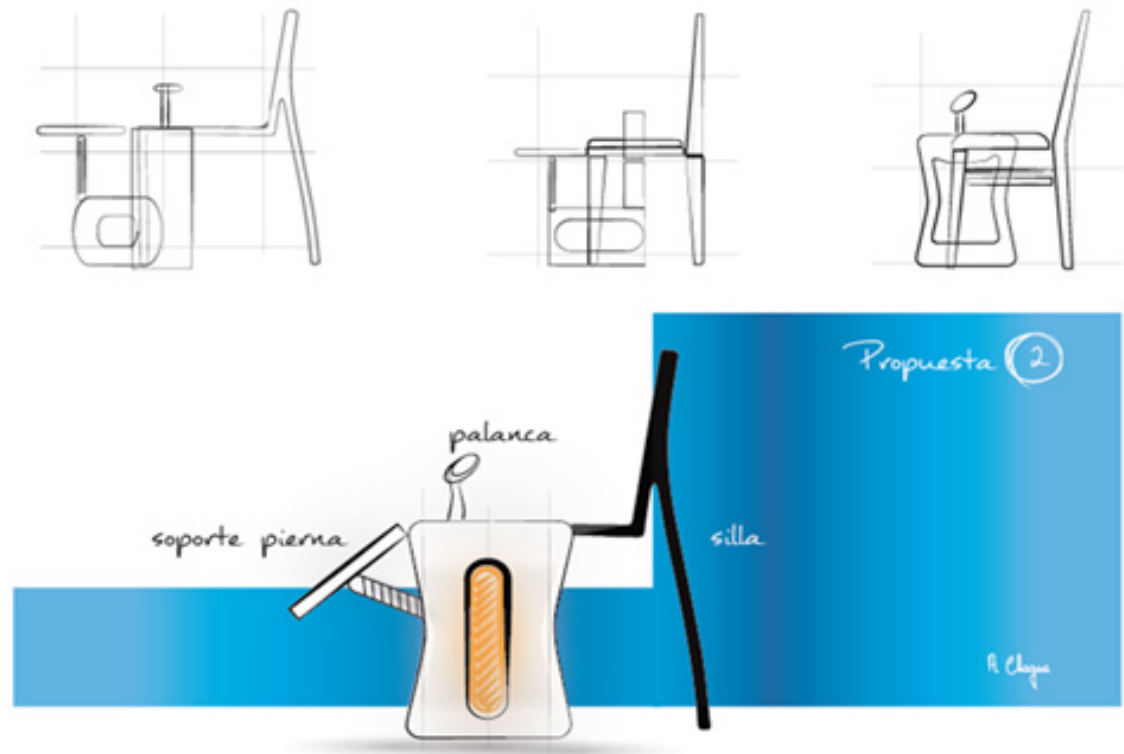

Figura 10. Proceso de diseño de la Forma 3
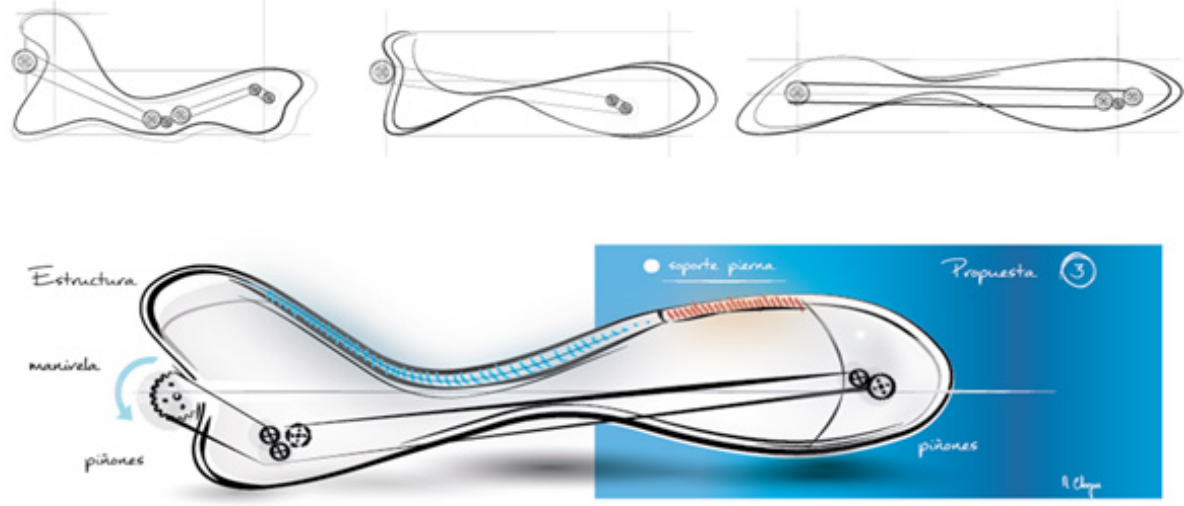

Figura 11. Proceso de diseño de la Forma 4
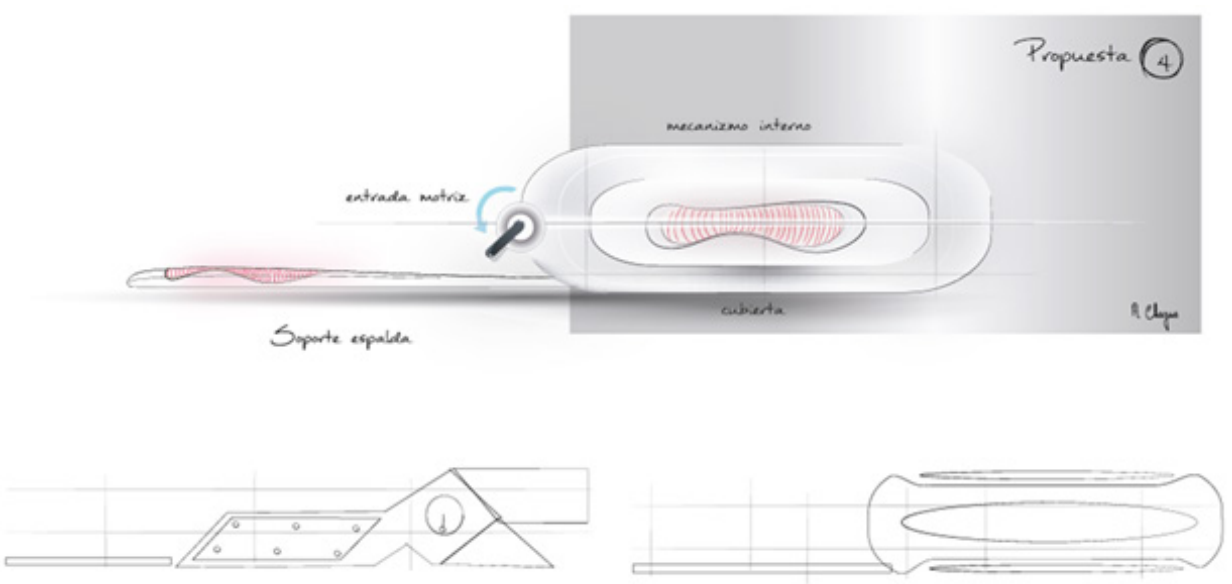
Figura 12. Proceso de diseño de la Forma 5

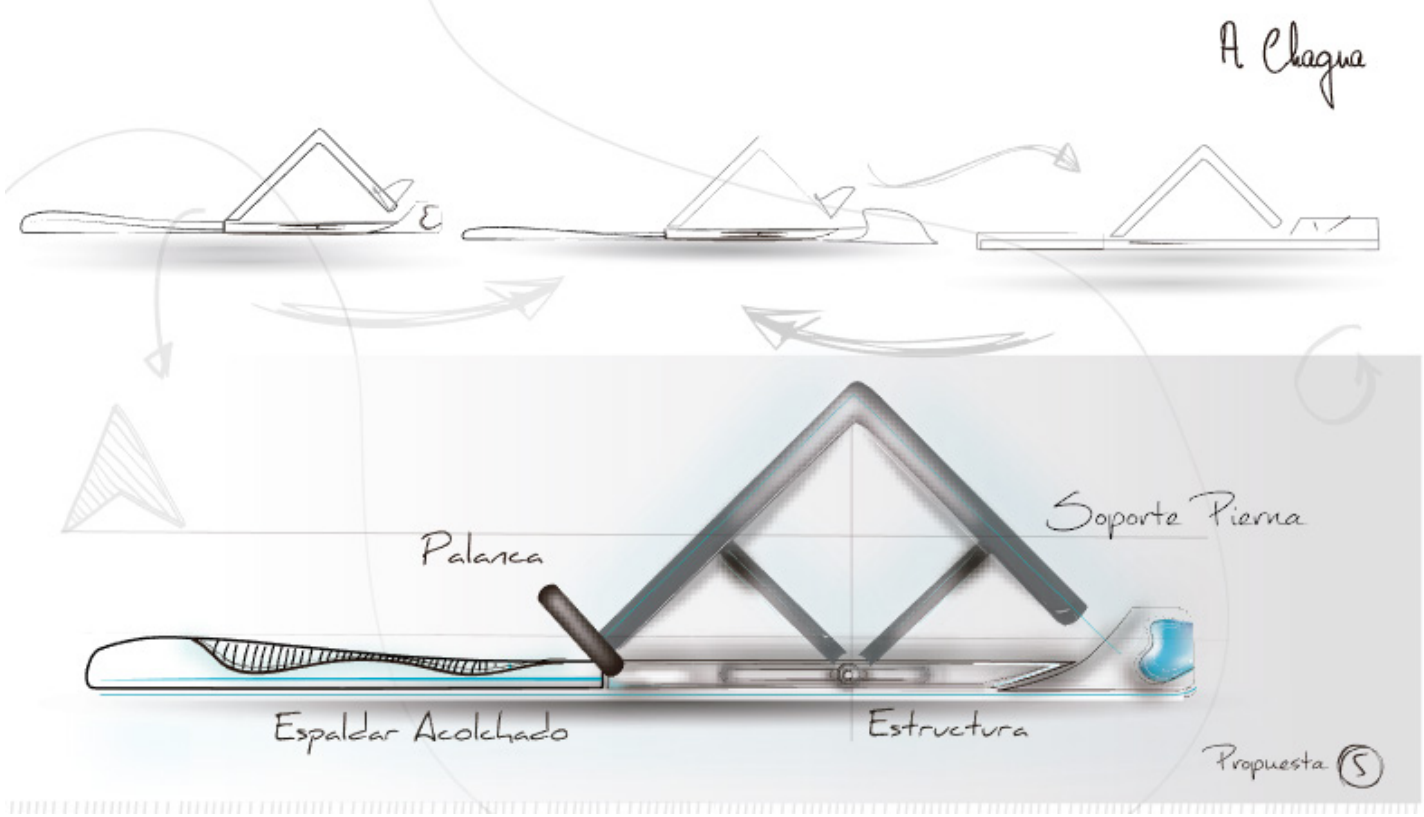

En la figura 13, se observa que la Forma 5, considerando los criterios de selección en forma global, resulta ser la más idónea y, además, cumple por encima de todas las alternativas con mayor puntaje en cada criterio.

Figura 13. Evaluación de las alternativas de la forma

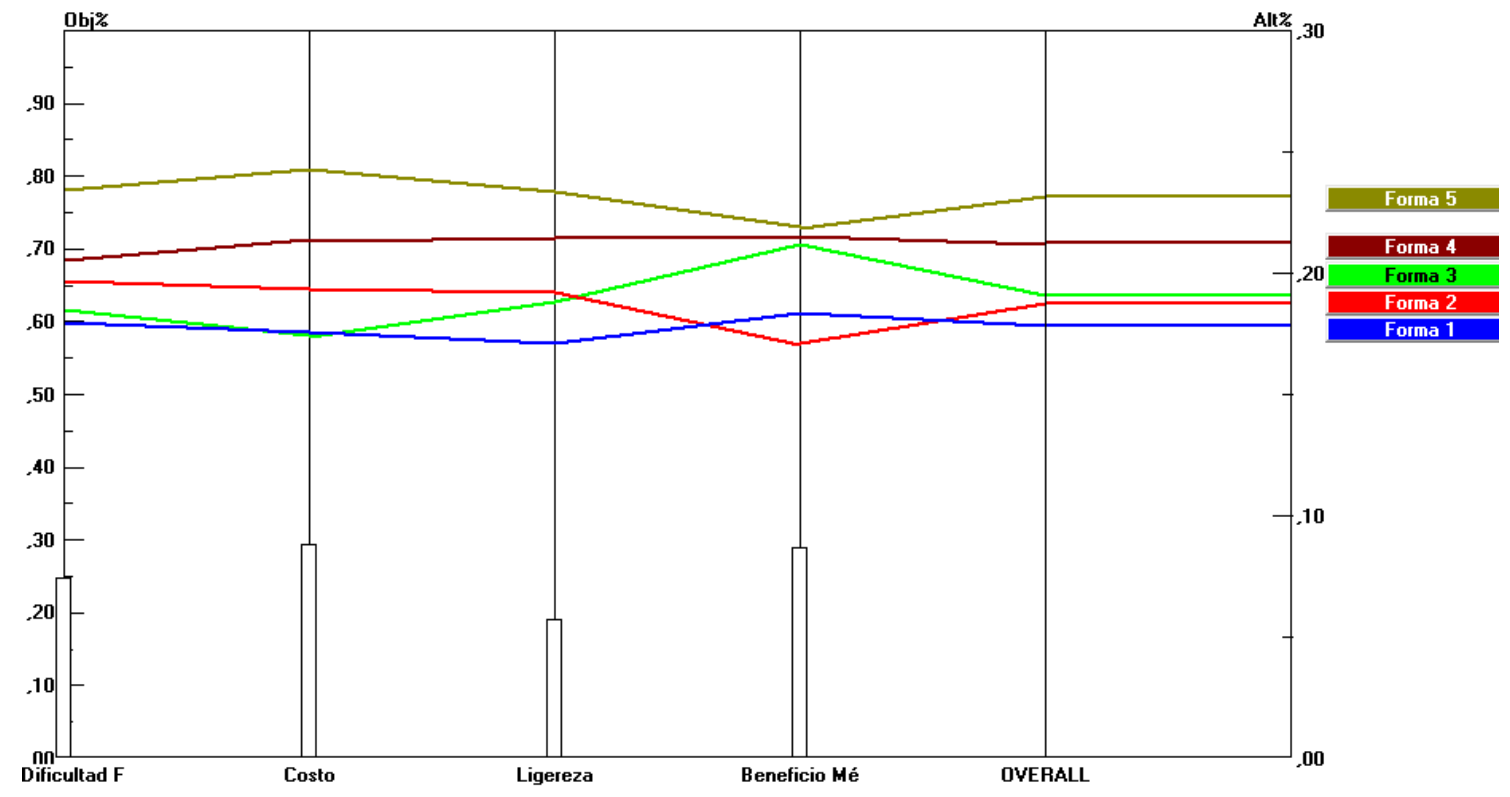


Por último, se presenta la sensibilidad global para seleccionar la materia prima de los componentes del rehabilitador en la figura 14. En ésta se muestra que, la opción del aluminio, puede ser más liviana y se acopla a las necesidades planteadas y queda por debajo del ABS y, por lo tanto, ambos materiales se pueden trabajar conjuntamente y llegar a cumplir funciones complementarias en el desarrollo del prototipo.

Figura 14. Evaluación de las alternativas para el material

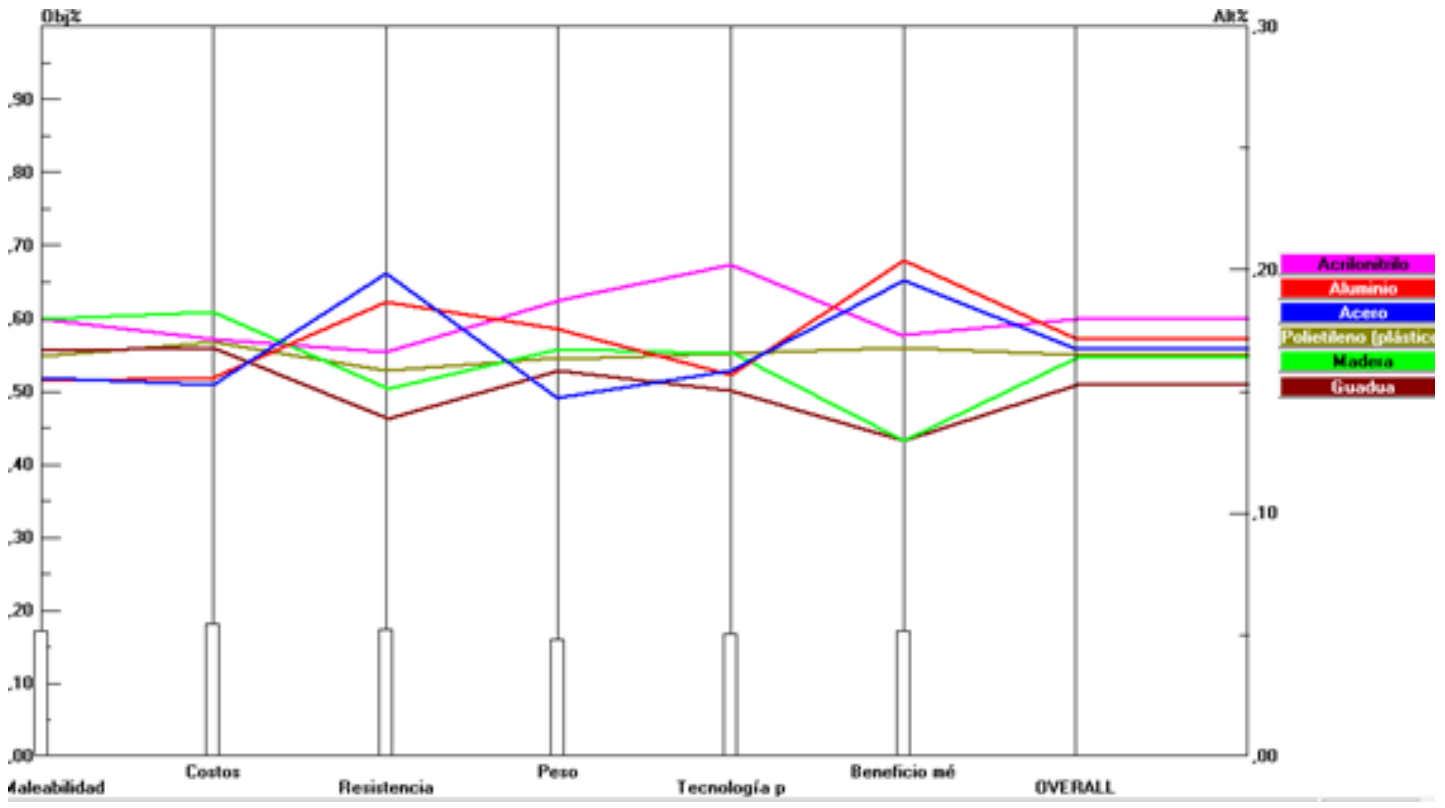

\section{Análisis Cinemático del mecanismo}

A través de la síntesis cinemática del mecanismo se establecen las dimensiones de los eslabones que lo conforman y la trayectoria que éstos describen, desarrollando una propuesta que cumple con los movimientos de flexión y extensión, propuestos para cada etapa de rehabilitación. Así, los eslabones del mecanismo, están asociados a las dimensiones del muslo y la pantorrilla respectivamente.

Es importante resaltar que la cinemática del dispositivo debe mantener una concordancia con las especificaciones de diseño asociados a las dimensiones de las extremidades inferiores y pesos según el usuario, establecidos en la antropometría, como se observa en la tabla 1 y se detalla en (Romero, 2012), lo que asegura que la posición de la rodilla del paciente coincida de forma análoga con los eslabones que transmiten el movimiento.

Tabla 1. Dimensiones y pesos considerados en el mecanismo

\begin{tabular}{|l|l|l|l|}
\hline \multicolumn{2}{|c|}{ Muslo } & Pierna & Pie \\
\hline Longitud (cm) & 41 & 41 & 25 \\
\hline Masa (kgf) & 12,00 & 5,58 & 0,17 \\
\hline
\end{tabular}

El dispositivo presentado en la figura 15 y simulado en working model, posee un sistema de barras que se activan cuando el actuador transmite una fuerza correspondiente a la aplicada por el usuario, permitiendo un desplazamiento proporcional, en donde interviene la parte de biela y carro que se encuentran ensamblados como un solo sistema. Las fuerzas en cada uno de los eslabones, que están detalladas en los pines, serán utilizadas para el análisis estructural. Todo el mecanismo está apoyado sobre una superficie que, además de servir de bancada en la geometría final, corresponde a un accesorio del rehabilitador 
Figura 15. Posición crítica del mecanismo simulado en working model

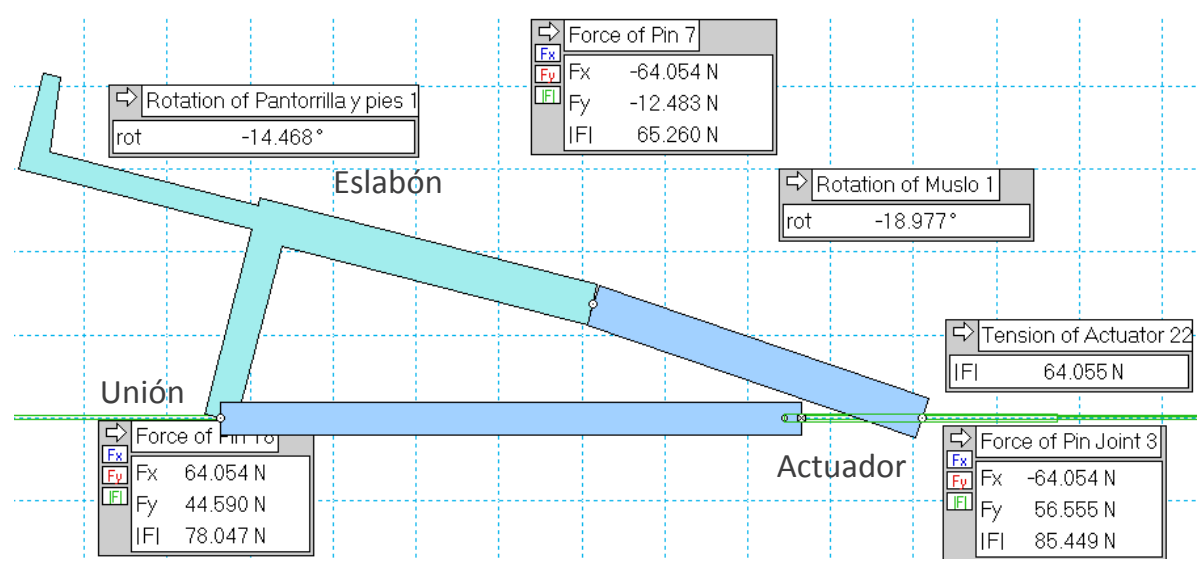

\section{Generación de la geometría. Propuesta con Autodesk Inventor}

Las figuras 16 y 17 muestran el resultado de la generación de la geometría en Inventor ${ }^{\circledR}$ para cada uno de los componentes que conforman el dispositivo de rehabilitación y en la tabla 2 se presenta una descripción de éstos.

El Rehabilitador de Rodilla presentado tiene un grado de libertad, donde en la fase inicial de la rehabilitación por cada grado que gire el mango (2), se desplaza el mismo ángulo en la rodilla (barras 11); mientras que esta relación es de 2:1 para la fase final de rehabilitación. El rehabilitador, se compone de 13 elementos que de acuerdo a su numeración corresponden: anclaje del rehabilitador (1), mango (2), articulación (3), uniones (4), soporte muslo (5), soporte pantorrilla (6), apoyo de pie (7), ajuste de longitud pantorrilla (8), corredera (9), base de corredera (10), barras apoya muslo y pantorrilla (11), manivela (12), biela (13). El ensamble global permite que el mecanismo manivela - biela - corredera tenga una variación de ángulos entre el muslo y la pantorrilla, que van de $170^{\circ}$ a $90^{\circ}$. Por lo tanto, el movimiento de flexión y extensión en la rodilla se desarrolla de acuerdo a la posición del mango (2) que es la entrada que activa el mecanismo de manivela (12), biela (13) y corredera (9), ensamblados de acuerdo a las articulaciones (3) y uniones (4). Todo el sistema de rehabilitación está unido a la bancada formada por el espaldar que es un elemento acolchado tipo accesorio (ver figura 17) unido a la base de la corredera (10) y el anclaje del rehabilitador de rodilla (1) por medio de un sistema de sujeción tipo velcro. El movimiento angular entre las barras (11) está condicionado por cada fase angular de recuperación, donde el movimiento se realiza a través del acoplamiento de seis barras con una sola entrada motriz, proporcionada por el usuario por (2). Este conjunto, define la extensión de la rodilla y su flexión en cualquier ángulo controlado de la rodilla, cuando se apoya el muslo en (5) y la pantorrilla en (6). Así, el peso de estas extremidades debe ser vencido por la única entrada proporcionada por (2). El rango de movimiento resultante es determinado por la corredera (9) con un movimiento lineal.

Figura 16. Geometría propuesta para el rehabilitador de Rodilla y sus partes
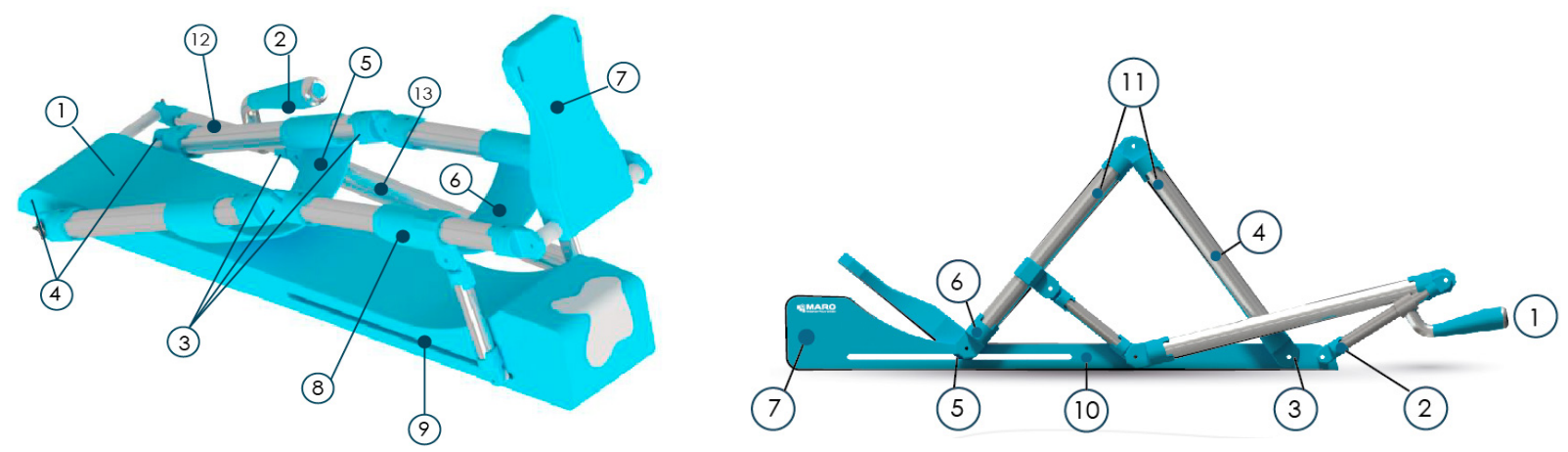
Figura 17. Base acolchada del rehabilitador

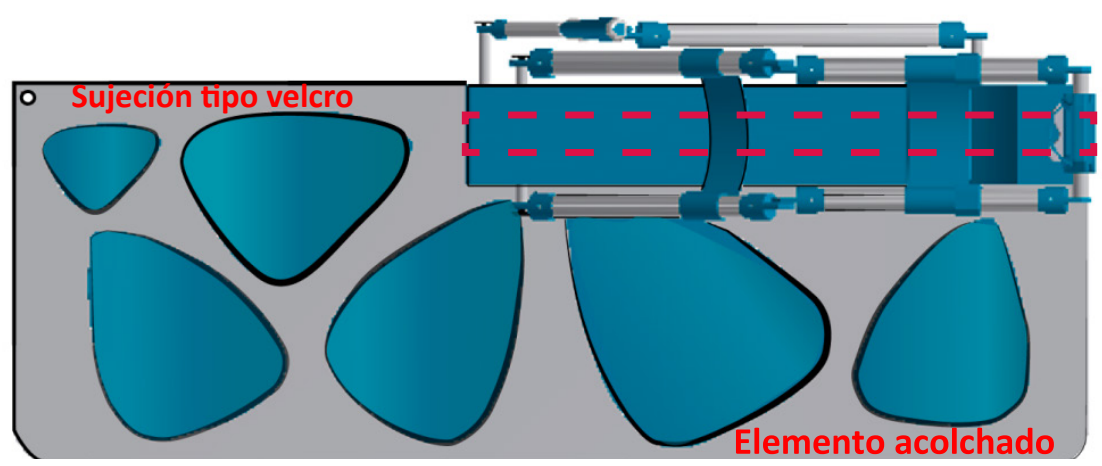

Tabla 2. Descripción de los elementos del rehabilitador de Rodilla

\begin{tabular}{|l|c|c|}
\hline Descripción & Elemento & Cantidad \\
\hline Anclaje del Rehabilitador & 1 & 1 \\
\hline Mango & 2 & 1 \\
\hline Articulación & 3 & 6 \\
\hline Uniones & 4 & 6 \\
\hline Soporte muslo & 5 & 1 \\
\hline Soporte pantorrilla & 6 & 1 \\
\hline Apoyo de pie & 7 & 1 \\
\hline Ajuste de longitud pantorrilla & 8 & 2 \\
\hline Corredera & 9 & 2 \\
\hline Base de corredera & 10 & 1 \\
\hline Barras apoya muslo y pantorrilla & 11 & 4 \\
\hline Manivela & 12 & 1 \\
\hline Biela & 13 & 1 \\
\hline
\end{tabular}

\section{Análisis Estructural}

El prototipo del rehabilitador de rodilla se puede construir de tubo de aluminio que presenta las propiedades mecánicas que cumplen con las solicitaciones de carga requeridas para que el mecanismo cumpla su función; además, los elementos para el ensamble podrán ser realizados con impresión 3D (ABS). El análisis estructural se realiza asumiendo que el aluminio es un material isotrópico, homogéneo y dúctil. La tabla 3 muestra las propiedades mecánicas y físicas de estos materiales, necesarias para desarrollar la simulación numérica.

Tabla 3. Propiedades Mecánicas del Aluminio y ABS

\begin{tabular}{|c|c|c|c|c|}
$\begin{array}{c}\text { Módulo de } \\
\text { Poisson }\end{array}$ & $\begin{array}{c}\text { Esfuerzo de } \\
\text { Fluencia (MPa) }\end{array}$ & $\begin{array}{c}\text { Esfuerzo último de } \\
\text { Tensión (MPa) }\end{array}$ & $\begin{array}{c}\text { Módulo de } \\
\text { Young (GPa) }\end{array}$ & $\begin{array}{c}\text { Densidad (g/ } \\
\mathbf{c m}^{\mathbf{3}} \mathbf{)}\end{array}$ \\
\hline 0,33 & 275 & 310 & 68,900 & 2,710 \\
\hline 0,38 & 20 & 29,6 & 2,240 & 1,060 \\
\hline
\end{tabular}




\section{Restricciones de movimiento}

Para el estudio del rehabilitador, se toma la posición crítica mostrada en la figura 15 que corresponde a los $170^{\circ}$ que forma el muslo con la pierna. Se colocó una restricción de tipo soporte fijo en el extremo más largo de la base, justo en la zona donde se apoya todo el mecanismo y que en la figura 18 se detalla.

Globalmente, en el rehabilitador se verifican 284 regiones de contacto, las cuales se han generado automáticamente y son del tipo de "no separación", suficientes para asegurar el ensamble del conjunto.

El análisis tensional del sistema se realiza para las cargas establecidas en la tabla 1 que representan el peso del muslo y la pantorrilla. Las direcciones de éstas, se definen de acuerdo al peso y sentido hacia dentro del dispositivo, de acuerdo a la transmisión de carga por los elementos que apoyan el muslo y la pantorrilla, como se observa en la figura 18.

Una vez definido todo el sistema para la simulación en Inventor, es decir, la geometría, el material, las restricciones y el estado de carga, se procede a realizar la simulación numérica.

Para obtener una solución con el método de elementos finitos, se sigue un algoritmo simple dentro del proceso del diseño (ver figura 2) y en el cuál, se contemplan los pasos requeridos. Para la simulación de análisis estructural estático, se utiliza la plataforma de Inventor con mallas refinadas de topologías tetraédricas que convergen hacia la solución con relación de convergencia menor al 5\%. Al obtener resultados donde la geometría y el material del sistema son capaces de soportar las cargas máximas, sin deformarse permanentemente, se precisa la geometría final para pasar al diseño de detalle.

Figura 18. Restricciones de movimiento y Fuerzas del rehabilitador.

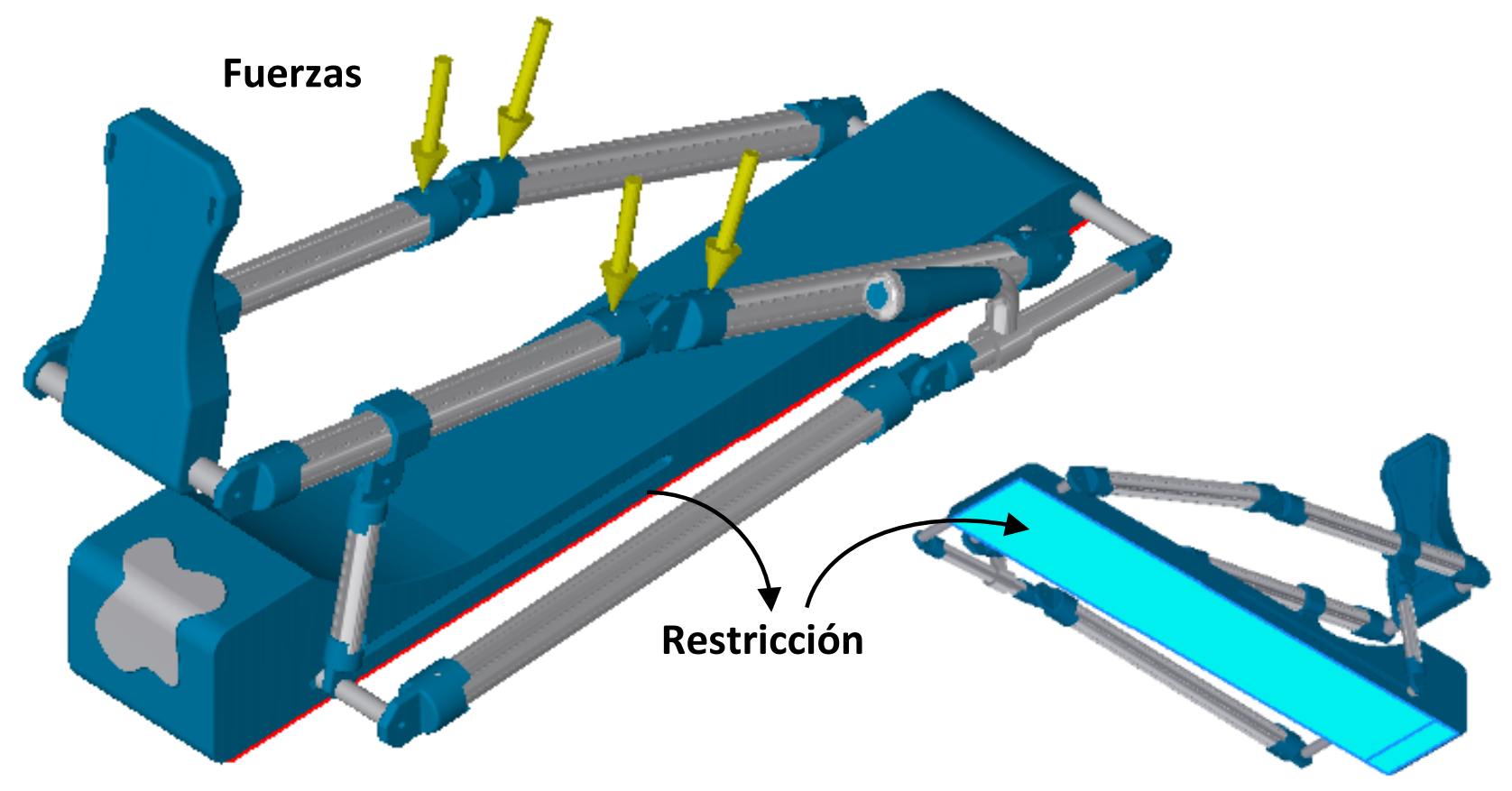

\section{Refinamiento de Malla para el análisis estático}

El punto de estudio, donde se presenta el esfuerzo máximo para cada refinamiento, corresponde a la unión de las articulaciones de las barras que soportan el muslo. Así, se realiza un mallado con refinamiento automático, compuesto por los elementos que se detallan en la tabla 4. En la figura 19 se muestra la última malla con el 
mejor refinamiento para obtener el comportamiento del rehabilitador, ante las condiciones máximas de carga asociadas a la resistencia del material, los desplazamientos, la deformación y el factor de seguridad.

Tabla 4. Tipo y características de los elementos que se emplean

\begin{tabular}{|l|c|c|c|}
\multirow{2}{*}{ ANÁLISIS } & \multicolumn{3}{|c|}{ ELEMENTOS SÓLIDOS } \\
\cline { 2 - 3 } & TIPO & CANTIDAD & TOTAL \\
\hline \multirow{2}{*}{ Rehabilitador de rodilla } & Tetraedros & 383413 & \multirow{2}{*}{383413} \\
\cline { 2 - 3 } & Nodos & 704854 & \\
\hline
\end{tabular}

Figura 19 Malla última en el refinamiento

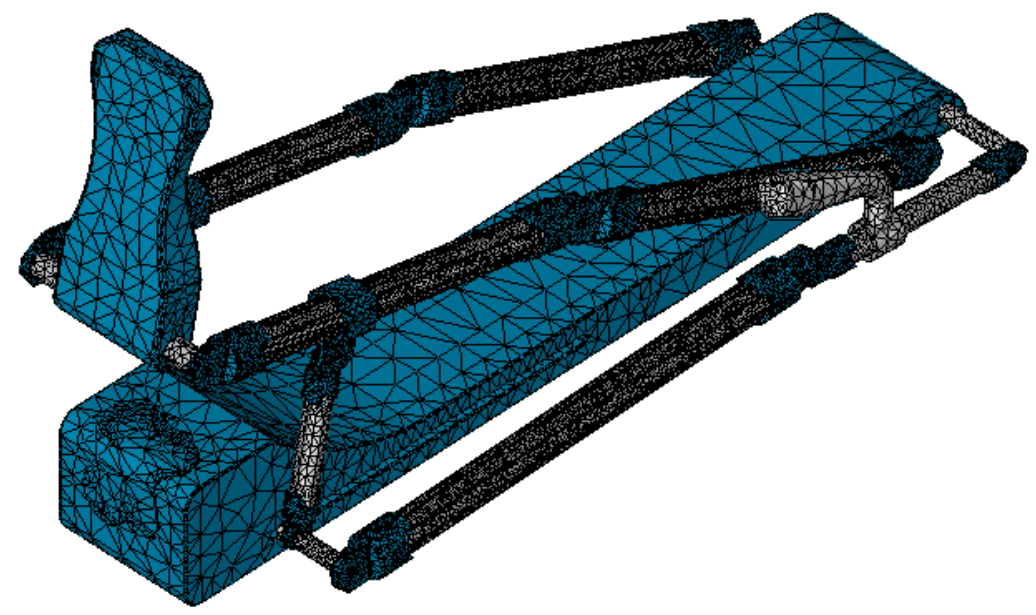

En las figuras 20 y 21 se muestra el esfuerzo equivalente de Von Misses en el último refinamiento de malla y el factor de seguridad estático, respectivamente. En estas, puede observarse que el esfuerzo producido por las cargas del muslo y de la pierna son menores que la resistencia del material ya que, éste no sobrepasa los $65 \mathrm{MPa}$; mientras que la resistencia de fluencia del aluminio es de $275 \mathrm{MPa}$. Por lo anterior, se puede verificar que el factor de seguridad presentado en la ecuación 1, es superior a la unidad y, por lo tanto, el rehabilitador no se deforma permanentemente y está lejos del límite de rotura del material.

Figura 20. Distribución de los esfuerzos de Von Misses

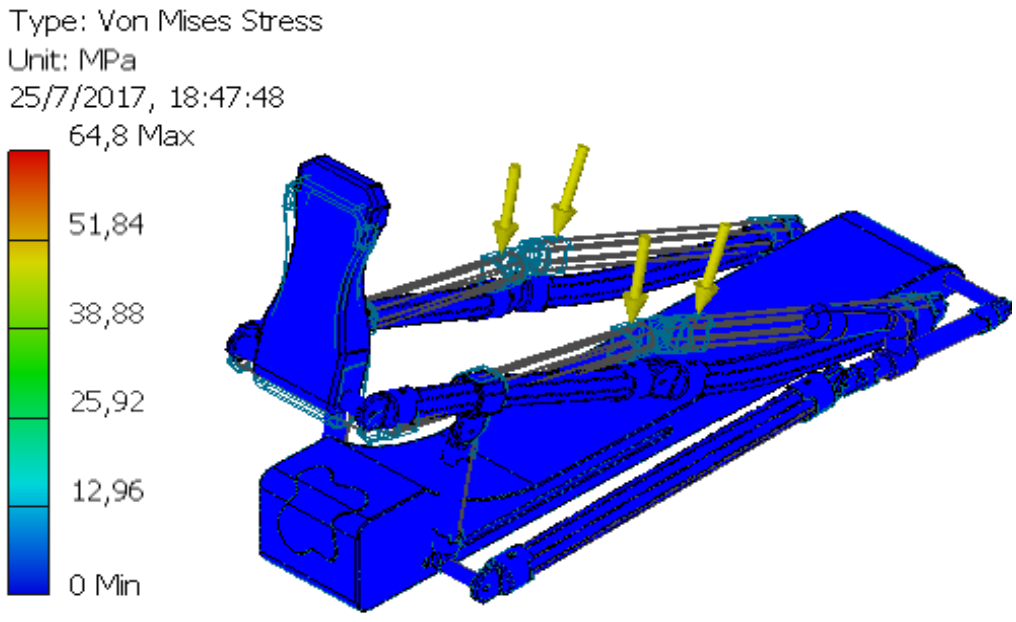


Figura 21. Factor de seguridad

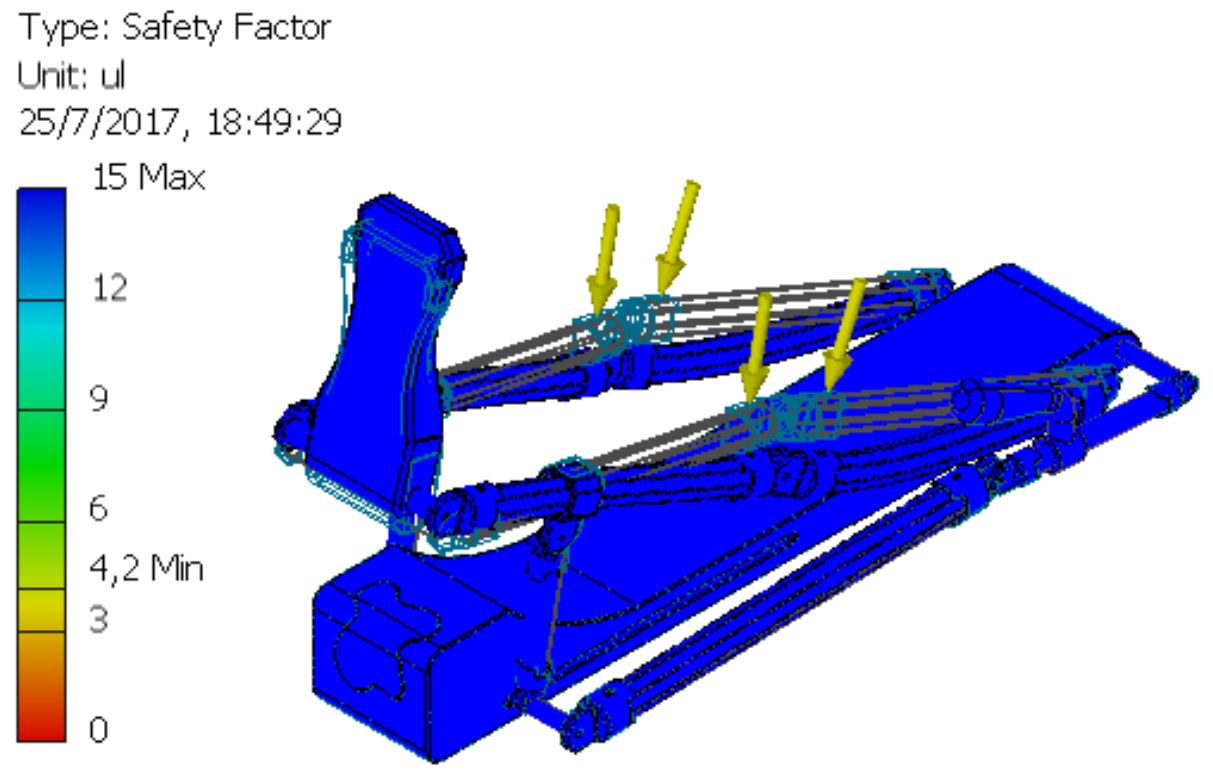

En la figura 22 se observa que los desplazamientos no superan el 0.06 milímetros; mientras que la figura 23 muestra que la deformación producida para las cargas máximas establecidas no sobrepasa el 1\%. Por lo anterior, se visualiza que, con estas magnitudes de trabajo, el rehabilitador no fallará y tendrá deformaciones pequeñas.

Figura 22. Distribución de las Desplazamientos

Type: Displacement

Unit: $\mathrm{mm}$

25/7/2017, $18: 48: 46$

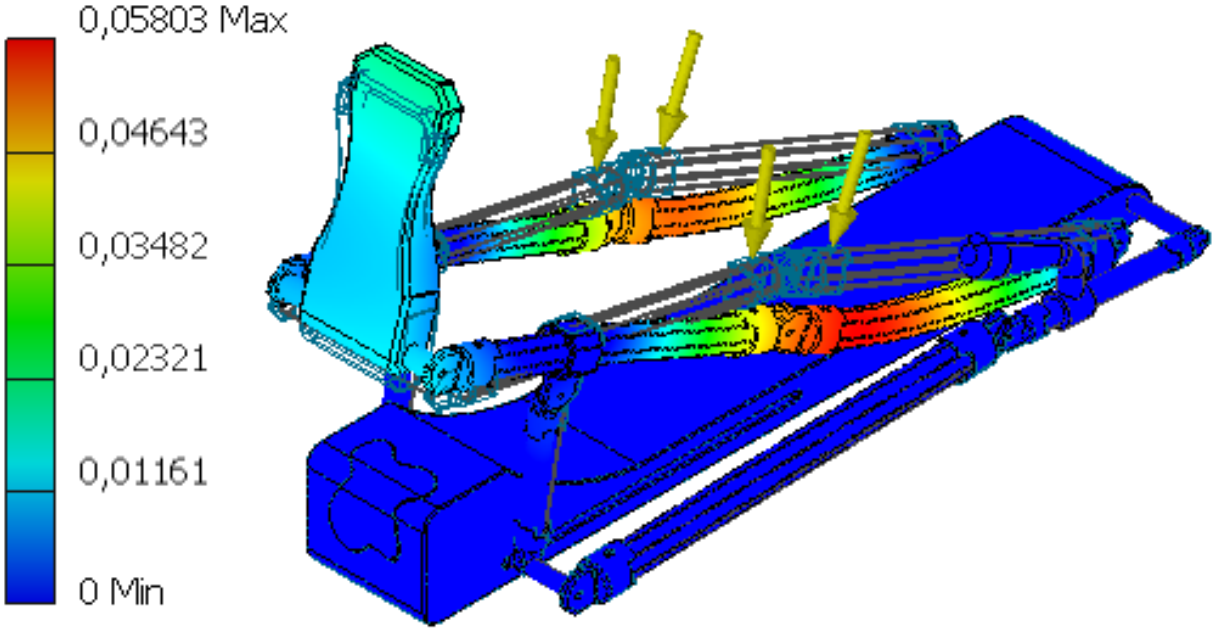


Figura 23. Distribución de las Deformaciones

Type: Equivalent Strain

Unit: ul

25/7/2017, 18:50:05

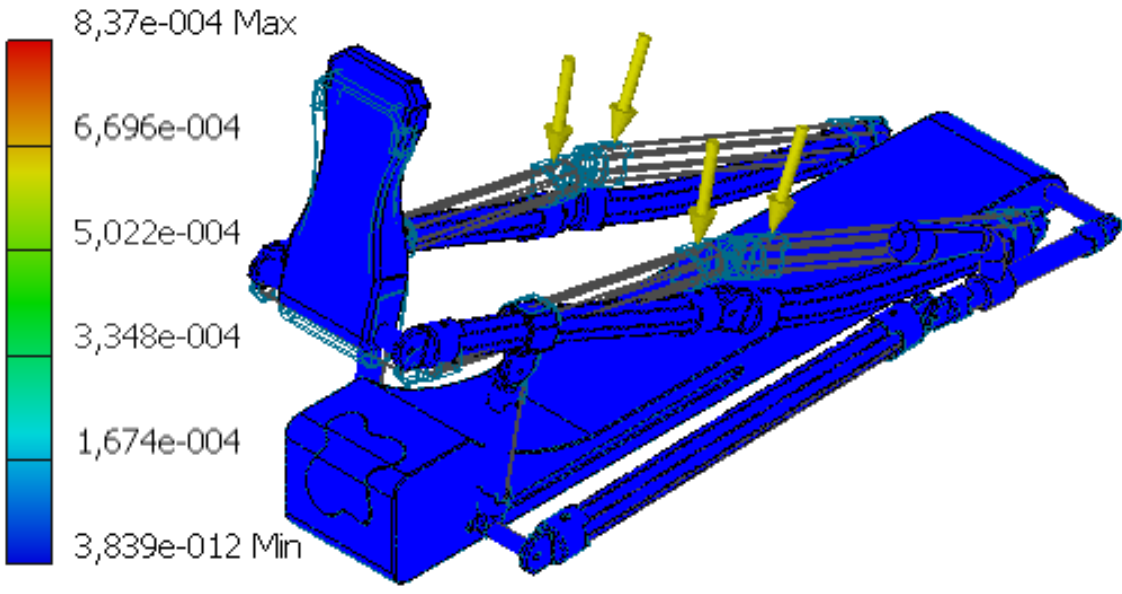

\section{Verificación numérica del dispositivo. Convergencia}

Para la verificación de la simulación realizada, se debe analizar la curva de convergencia de los esfuerzos de Von-Mises hacia la solución. En la figura 24, se muestra que se realizaron 23 refinamientos, de los cuales a partir del 19 converge hacia el esfuerzo máximo de 64,8 MPa, lo que define que éste es el valor esperado según las cargas introducidas por el muslo y la pierna en el rehabilitador, con una rata de convergencia inferior al 0,006\%.

Figura 24. Convergencia de los esfuerzos máximos

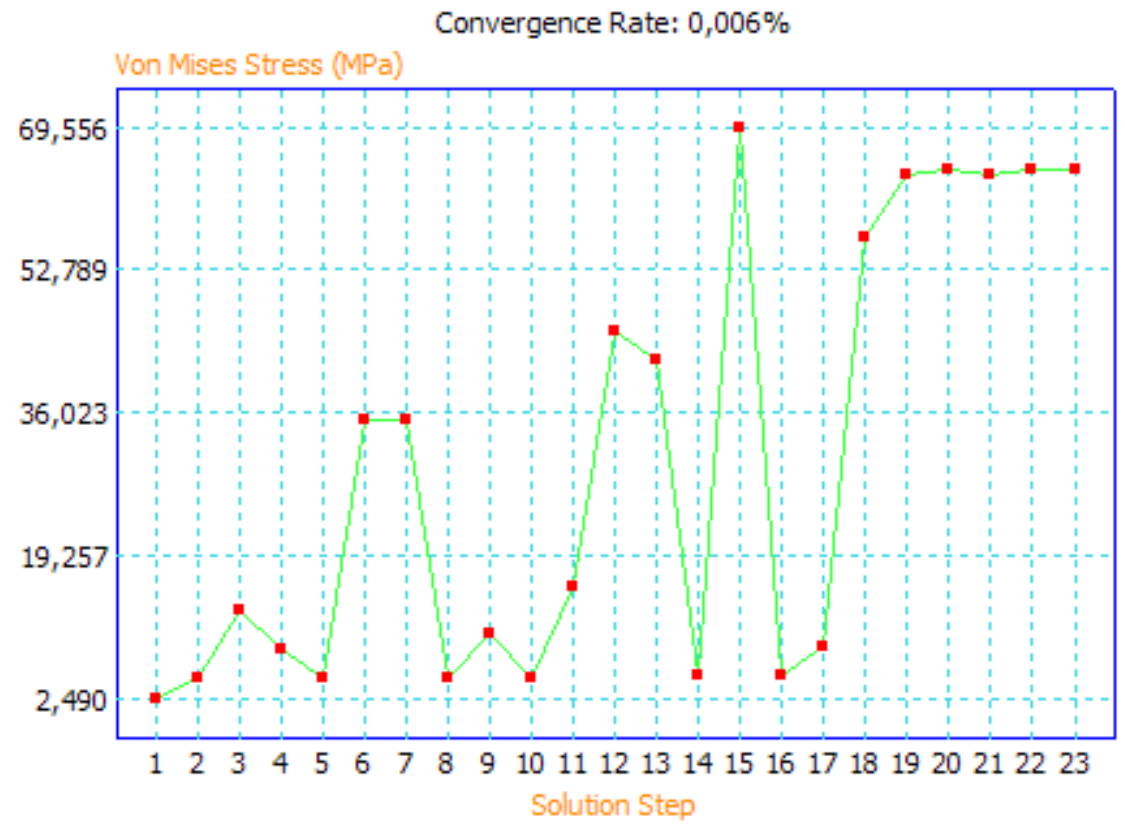




\section{CONCLUSIONES}

Mediante el diseño propuesto se realizó un proceso de diseño del prototipo del dispositivo para rehabilitación de la rodilla. El proceso seguido consideró la evaluación y selección de posibles alternativas de solución para el sistema de accionamiento y material, el modelado y documentación de la geometría del dispositivo de rehabilitación en un paquete $\mathrm{CAD}$, la realización del estudio cinemático con la ayuda de herramientas CAE y el análisis estructural del dispositivo, en base a simulaciones hechas en Inventor. Se ha desarrollado una propuesta de diseño de un dispositivo de rehabilitación pasiva de rodilla, que cumple con movimientos de flexión y extensión, basado en un mecanismo de cuatro barras con un grado de libertad, con materiales disponibles en el mercado nacional.

Los resultados del análisis estructural obtenidos para la distribución del esfuerzo y el factor de seguridad, muestran que la geometría planteada, basándose en la posición crítica, es decir donde la absorción de carga sobre el mecanismo es más crítica, cumplen con los requisitos preestablecidos, donde el rehabilitador no se deformara permanentemente y los desplazamientos son menores al $0.06 \mathrm{~mm}$. Por lo tanto, el dispositivo de rehabilitación de rodilla no fallará desde el punto de vista de resistencia y de deflexión del material.Se realizó un estudio de convergencia y error, encontrándose que los valores convergen y representan un porcentaje de error menor al 5\%, lo cual refleja la precisión del estudio realizado y, a la vez, hace válida la simulación para el análisis con carga estática.

\section{Agradecimiento}

La presente investigación agradece al Centro de Investigaciones de la Pontificia Universidad Católica del Ecuador Sede Ibarra por su aporte al desarrollo de la misma.

\section{REFERENCIAS BIBLIOGRÁFICAS}

Archer, B. (2015) Metodología de diseño. Importancia de su aplicación en las disciplinas proyectuales. Argentina Publicaciones DC. Recuperado de http://fido.palermo.edu/servicios_dyc/publicacionesdc/ vista/detalle_articulo.php?id_articulo=30\&id_libro=7

Branch, T, Cunningham, T., Dittmar, E., y Jacobs, C. (2016). Robotic Knee Testing Device, Subjective Patient Input Device and Method for using same. United States. US2016338649 (A1). Patente Internacional.

CONADIS. (2016). Consejo Nacional para la Igualdad de Discapacidades. Informe Estadístico de Personas con Discapacidad. Quito, Ecuador: Ministerio de Salud Pública de Ecuador. Recuperado de http:// www.consejo discapacidades. gob.ec/

Ding, X., Yang, T., Qian, F., Ding, J., y Zhao, Z. (2016). Knee joint flexion function rehabilitation exercise device. China. CN20161 308652.Patente Internacional.

Guzmán Valdivia, C. H., Blanco Ortega, A., Oliver Salazar, M. A., y Azcaray Rivera,H. R. (2014). Modelado y Simulación de un Robot Terapéutico para la Rehabilitación

Hall J. (2017). Knee Rehabilitation Device. United States. US. 2017027799. Promotus LIc. Patente Internacional.

INEC. (2016). Instituto Nacional de Estadísticas y Censos. Anuario de Estadísticas Hospitalarias: Egresos y Camas. Quito, Ecuador: Dirección de estadísticas sociodemográficas. Recuperado de: http://www. ecuador en cifras.gob.ec/camas-y-egresos-hospitalarios/

Rashid, K. (2017). Karimanifesto Recuperado de http://www.karimrashid.com/karimanifesto

Romero, M. (2012). Diseño y construcción de una ortesis de rodilla, destinada a la rehabilitación automatizada de la extremidad inferior. Tesis de pregrado. Universidad Politécnica Salesiana. Cuenca, Ecuador.

Schmitt, C., y Métrailler, P. (2004). The Motion MakerTM: a rehabilitation system combining an orthosis with closed-loop electrical muscle stimulation. En 8th Vienna International Workshop on Functional Electrical Stimulation, Viena, Austria.

Swortec. (2017). MotionMaker ${ }^{\mathrm{TM}}$. Recuperado de http://www.swortec.ch/index.php/products/motionmaker

Tian, F., Hefzy, M. S., y Elahinia, M. (2015). State of the Art Review of Knee-Ankle-Foot Orthoses. Annals of biomedical engineering, (43)(2), 427-441. 
Umchid, S., y Taraphongphan, P. (2016). Design and development of a smart continuous passive motion device for knee rehabilitation. In Biomedical Engineering International Conference (BMEiCON), 2016 9th (pp. 1-5). IEEE.

Vergara, M., Segnini, J., Santacruz, P., y Kastillo, A. (2017). Diseño de un bipedestador modular pediátrico que incorpora el arte precolombino Ecuatoriano. Vergara, M., Díaz, M., Rivas, F. y Restrepo, M. Diseño de Equipos de Rehabilitación y Órtesis.

Vergara, M., Segnini, J., Provenzano, S., Chagna, A., y Diez, J. (2017). Diseño de un Dispositivo para Autorehabilitación Pasiva de Rodilla no Supervisada. Vergara, M., Díaz, M., Rivas, F. y Restrepo, M. Diseño de Equipos de Rehabilitación y Órtesis.

Wilkening, A., Baiden, D., y Ivlev, O. (2012). Assistive acting movement therapy devices with pneumatic rotary-type soft actuators. Biomedizinische Technik/Biomedical Engineering, (57)(6), 445-456.

Xiaoning, L., Jianping, L., Zhongsheng, S., Yan, T., y Gang, Y. (2010). Flexible active and passive kneejoint rehabilitation training device. China. CN 201010146319. Univ. Nanjing Science \& Tech. Patente Internacional. 